\title{
All-Optical Graphene-Based Modulation of Surface Plasmon Polaritons via Modulation Instability for Secure Optical Communication
}

\author{
Morteza Abdolahi Sharif ${ }^{*}$ \\ Photonics Lab. Faculty of electrical engineering, Urmia University of Technology, Band road, \\ Urmia, Iran \\ *Corresponding Author Email:m.abdolahisharif@ee.uut.ac.ir
}

Regular paper: Received: Nov. 30, 2020, Revised: Jan. 16, 2021, Accepted: Feb. 23, 2021, Available Online: Feb. 25, 2021, DOI: 10.29252/ijop.14.2.177

\begin{abstract}
In this paper, an all optical graphene-based modulation approach is proposed induced by Modulation Instability (MI). The device structure is based on graphene sheets transferred on the both arms of a MachZehnder interferometer to support amplified Surface Plasmon Polaritons (SPPs). Due to the nonlinear nature of MI to interfere in the modulation process, the proposed approach leads to an enhanced performance in comparison to the conventional Mach-Zehnder modulators; using a low power cw driving beam $(\sim 20 \mu \mathrm{W}$ at $\lambda=50 \mu \mathrm{m})$, a high speed modulation rate ( $\sim 2$ Tpps) and subsequently, a high depth $(89 \%)$, wideband modulation $(\sim 81 \mathrm{GHz})$ can be resulted. Since the MI is a pre-state to the chaotic regime, the modulator can be also used for secure optical communication.
\end{abstract}

KEYWORDS: All-optical modulation, Graphene, Modulation instability, Optical amplification, Surface plasmon polariton.

\section{I.INTRODUCTION}

Optically driven systems present a nonlinear response beyond a threshold intensity. Meanwhile, for further increase in the intensity, a saturation of the nonlinear response is to be generally anticipated. However, those interacting with a feedback entity show a different sequential dynamical behavior including a primary ascending nonlinear response, bistability, Modulation Instability (MI) and transition to chaos [1-7]. MI is the consequence of the unbalanced competition between the nonlinearity and dispersion/diffraction. It appears through a quasi-periodic state (convective MI) and then progresses within a growing amplitude (absolute MI), a prior state to the chaotic regime [3-7]. MI seems to be undesirable but can on the other hand be useful for applications in supercontinuum generation [810], sideband generation [11], frequency comb generation [12-14] and optical modulation [1516].

Graphene is a 2D material with honeycomb lattice. It shows excellent electrical/optical properties including the high charge carrier mobility [17-18] and electrical/optical band gap tunability [19-20]. Due to its large effective surface, graphene can efficiently support Surface Plasmon Polaritons (SPPs). On this base, several studies represented the proof of concept for designing and fabricating the graphene-based optical modulators from electro-optical to all-optical plasmonic modulators [16, 23-37]. A huge nonlinearity can be released in a system containing the multiple graphene sheets embedded inside a dielectric host [21-22]. Herein, the absolute MI can be dominant driving by even a low threshold optical intensity. Utilization of the graphene huge nonlinear response in the modulation of SPPs may promise appealing issues not has yet been highly contemplated.

In our previous study, we proposed a novel graphene-based electro-optical modulator using the MI [16]. The enhanced output, fast 
modulation rate and high modulation depth were deduced as the main advantages. In this study, we take a step forward to introduce an all-optical graphene-based SPPs modulator using the MI. In addition, the proposed modulator has a hybrid working mechanism acting as the both electro-optical and alloptical modulator. The device utilizes a MachZehnder interferometer in order to provide a large feedback strength required for an alloptical modulation process. Indebted to the modern preparation and transfer methods [3840], the device can be fabricated in an ultrasmall size. The main difference with those previously presented devices is interfering the large nonlinear phase shift in the modulation process providing a broad band, high speed modulation and serving to amplification of SPPs. Furthermore, since the optical conductivity of graphene is tunable, the device nonlinear response can be adjusted at the absolute MI state, a prior one to chaotic regime, suggesting it for the secure optical communication.

\section{CONFIGURATION AND WORKING MECHANISM}

\section{A. Device Configuration}

Fig. 1 shows the schematic illustration of the proposed device configuration. The modulator is based on a subwavelength waveguide driven by a cw TM polarized beam with the wavelength, $\lambda=50 \mu \mathrm{m}$. The longitudinal size of the whole structure is $90 \mu \mathrm{m}$. The turn-on power is estimated to be about $\sim 20 \mu \mathrm{W}$ to push the device toward the absolute MI. The device uses the conventional silicon waveguide coupled with a graphene-based waveguide containing eight single-layer graphene sheets embedded inside a DEMETFSI ionic gel. The separation between each layer is $12.5 \mathrm{~nm}$. The assumption is in agreement with the maximum efficiency needed for the third order nonlinear response [22]. The whole structure is transferred on a $12.5 \mathrm{~nm}$ silica substrate. To work in a hybrid regime, the device uses Au electrodes in order to tune the graphene Fermi energy as well as to accelerate the charge carriers for the maximum attainable modulation speed. The dependence of the Fermi energy $E_{F}$ on the gate voltage $V_{g}$ can be given by [41]:

$$
E_{F}=\hbar v_{F}\left(\pi\left(n_{0}+C\left|V_{g}\right| / q\right)\right)^{1 / 2}
$$

where $v_{F}$ is the Fermi velocity; $\hbar$ is the reduced Planck's constant; $n_{0}$ is the intrinsic carrier density; $C$ is the effective capacitance per unit area; $V_{g}$ is the gate voltage and $q$ is the electric charge. Moreover, the device utilizes a Mach-Zehnder interferometer which warrantees a required feedback strength and also provides a constructive/destructive interference pattern required for all-optical modulation feature.

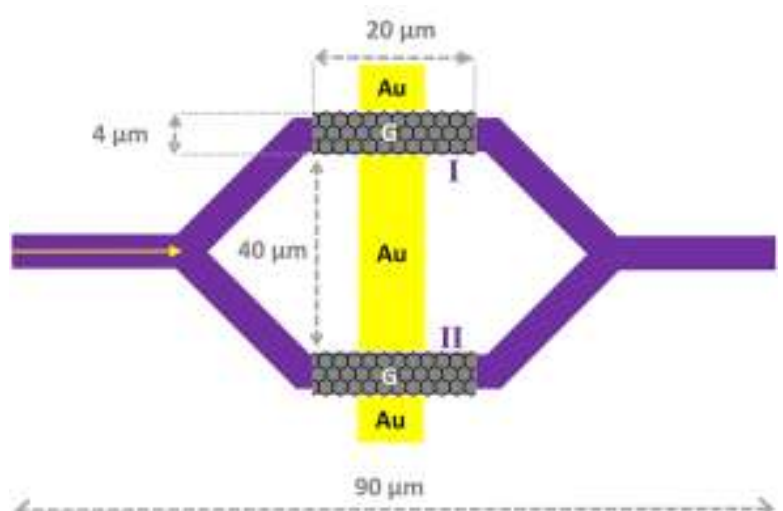

Fig. 1. Schematic illustration of proposed device; $G$ denotes embedded graphene sheet, Au means the $\mathrm{Au}$ electrodes. I and II denote arm I and arm II respectively.

\section{B. Theory and Simulation}

Spatial/temporal SPPs evolution in the absence of damping can be obtained by the universal spatial/spatiotemporal nonlinear amplitude equations (set of Eq. (2)) [1,42].

$$
\left\{\begin{array}{l}
2 i \frac{\omega}{c} \frac{\partial A}{\partial z}+\frac{\partial^{2} A}{\partial x^{2}}=-3 \chi^{(3)} \frac{\omega^{2}}{c^{2}}|A|^{2} A \\
\frac{\partial A}{\partial z}+\frac{1}{2} i k_{2} \frac{\partial^{2} A}{\partial t^{2}}=i \gamma|A|^{2} A
\end{array}\right.
$$

where $A$ is the slowly varying amplitude; $x$ and $z$ are the transversal and longitudinal propagation coordinates; $\omega$ is the frequency; $c$ is the light speed in vacuum; $k_{2}$ is the group 
velocity dispersion parameter; $\gamma$ is the nonlinear coefficient assigned to the graphenebased waveguide and $\chi^{(1)}$ and $\chi^{(3)}$ are the linear and third order graphene susceptibilities, measured in terms of the linear $\sigma_{1}$ and third order $\sigma_{3}$ optical conductivities. $\sigma_{1}$ can be given by Eq. (3) for interband and intraband transitions:

$$
\left\{\begin{aligned}
\sigma_{1, \text { inter }}= & \frac{i e^{2}}{4 \pi \hbar} \ln \left|\frac{2 E_{F}-\left(\omega+i \tau^{-1}\right) \hbar}{2 E_{F}+\left(\omega+i \tau^{-1}\right) \hbar}\right| \\
\sigma_{1, \text { intra }}= & \frac{i e^{2} k_{B} T}{\pi \hbar^{2}(\omega+i / \tau)} \times \\
& \left\{\frac{E_{F}}{k_{B} T}+2 \ln \left[\exp \left(-\frac{E_{F}}{k_{B} T}+1\right)\right]\right\}
\end{aligned}\right.
$$

where $e$ is the electron charge; $\tau$ is the relaxation time; $k_{B}$ is the Boltzmann constant and $T$ is the temperature [43-45]. In the same manner, $\sigma_{3}$ can be given by Eq. (4) for intraband transitions [43-45].

$\sigma_{3, \text { intra }}=-\frac{9}{8} i\left(\frac{e^{4} v_{F}^{2}}{\pi \hbar^{2} \omega^{3} E_{F}}\right)$

Mathematically, a perturbation in the solutions of Eq. (2) can result in an ultrafast quasiperiodic oscillations inferred as MI. A Fourier analysis of the oscillations can reveal that the sidebands number and amplitude gradually grow in the frequency domain. This can physically occur when the wave propagates inside a gain nonlinear media (here, the graphene-based waveguide). Beyond a critical frequency - which can be fulfilled if the nonlinearity or driving amplitude is greater than a threshold value - the character of MI will change from the convective to absolute. In this condition, the wave amplitude can grow even faster. Accordingly, absolute MI is often considered as a transitional state to the chaotic regime in the nonlinear optical systems [4647].

A quantity - known as the feedback strength $\rho$ - is introduced to describe the strength of interfering the waves in the two arms of Mach-
Zehnder interferometer and lies between 0 and $1[47]$.

\section{III.RESULTS AND DISCUSSION}

Two cases are assumed for comparison. The Energy Fermi for one arm (arm I) in MachZehnder interferometer remains constant at $E_{F}=0.4 \mathrm{eV}$ while that of the other arm (arm II) changes from $E_{F}=0.26 \mathrm{eV}$ (Case I) to $E_{F}=0.49 \mathrm{eV}$ (Case II). In each case, the main purpose is to interfere the waves of the two arms with different nonlinear phases. In addition, the feedback strength of the interferometer is assumed to change from $\rho=0.83$ (Case I) to $\rho=0.73$ (Case II). The results of the interference are then obtained for the assumed cases as follows.
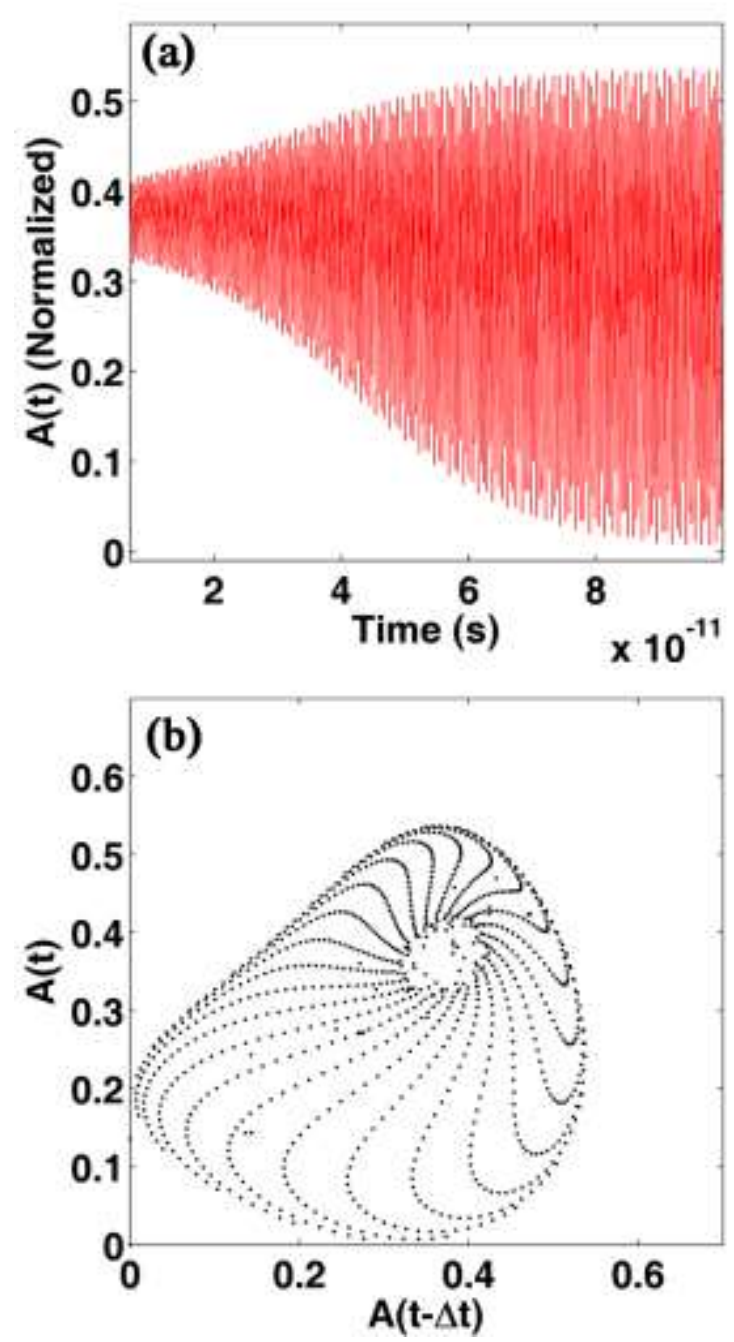

Fig. 2. (a) Time evolution and (b) phase portrait for $\mathrm{E}_{\mathrm{F}}=0.4 \mathrm{eV}$ in arm I of Mach-Zehnder interferometer. 


\section{A. Temporal dynamics}

Fig. 2(a) shows the temporal evolution of the wave amplitude in arm I which is distinguished as the convective MI. This can also be deduced from the phase portrait as in the form of a stable limit cycle shown in Fig. 2(b).

On the other hand, Fig. 3(a) shows the time evolution of the wave amplitude for the case I, obtained for the arm II. Due to the large nonlinearity - resulting from the assumed value for Fermi energy - the dynamical behavior is interpreted as the absolute MI which can be also seen from its phase portrait (Fig. 3(b)) as an attractor.
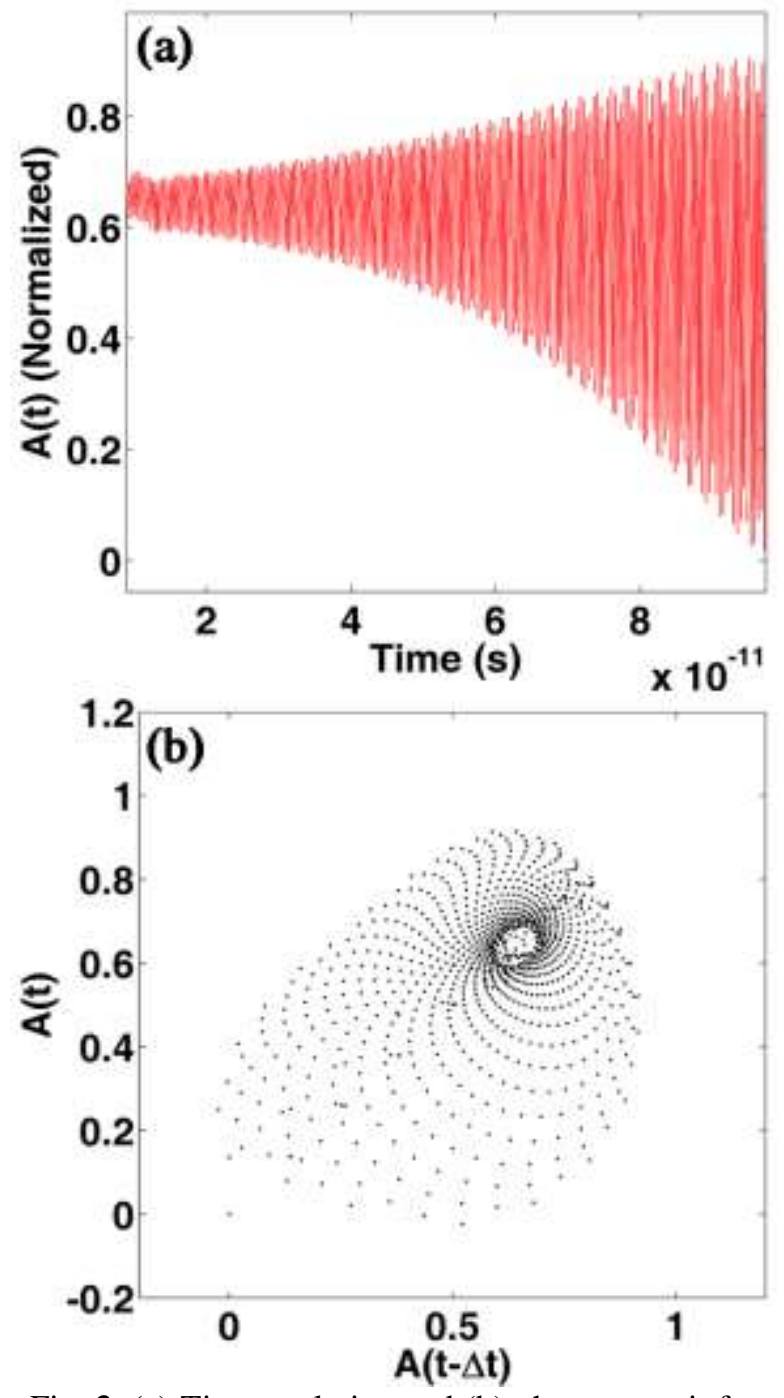

Fig. 3. (a) Time evolution and (b) phase portrait for $\mathrm{E}_{\mathrm{F}}=0.26 \mathrm{eV}$ in arm II (case I) of Mach-Zehnder interferometer.
In the next step, temporal evolution of the wave amplitude in arm II is obtained for the case II. This stands for a convective MI as shown in Fig. 4(a) and stressed in Fig. 4(b) through a neutrally stable limit cycle.

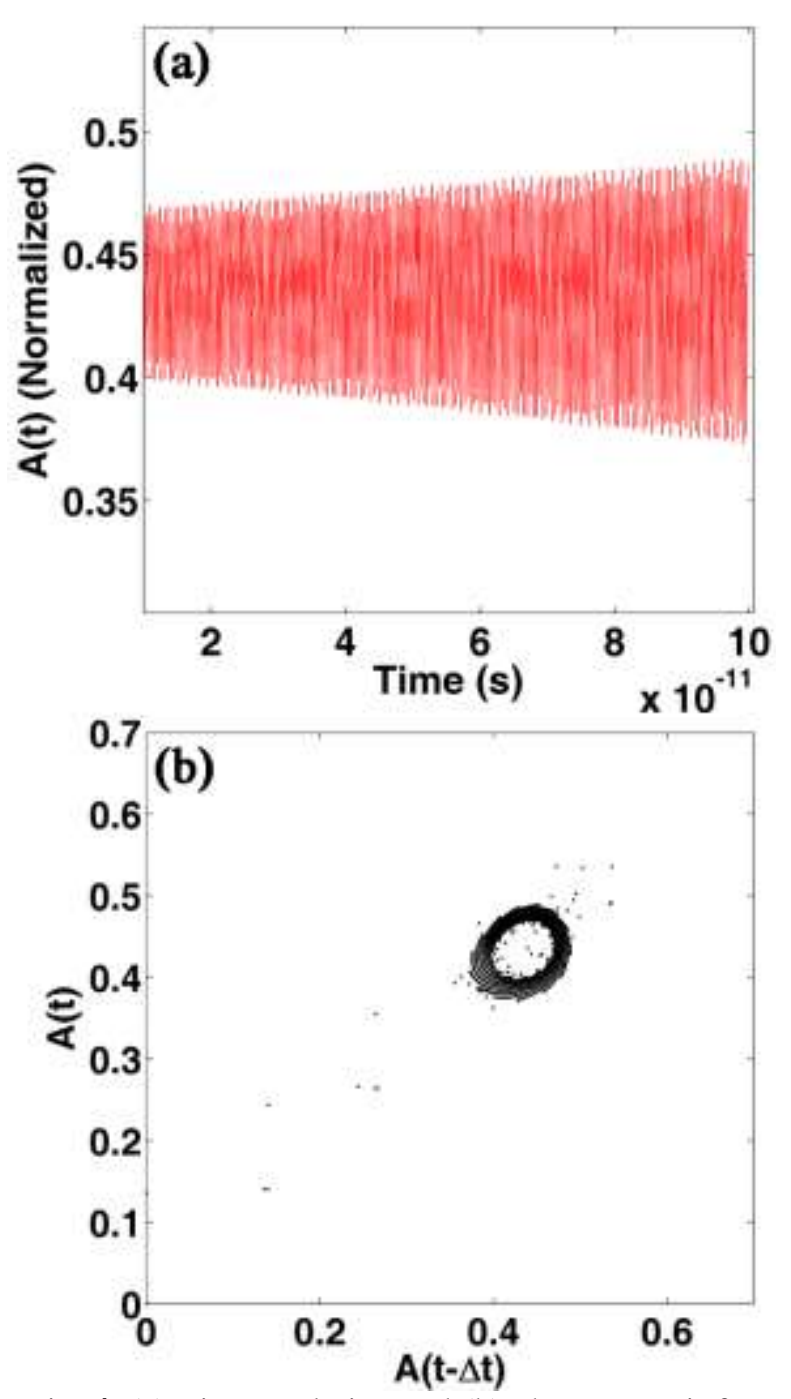

Fig. 4. (a) Time evolution and (b) phase portrait for $\mathrm{E}_{\mathrm{F}}=0.49 \mathrm{eV}$ in arm II (case II) of Mach-Zehnder interferometer.

The interference in the two arms appears in Fig. 5. The result for the case $I$ is shown in Fig. 5(a), while Fig. 5(b) shows the result for the case II. The interference waveform clearly includes an amplitude modulation for both cases. However, this interference arises from the nonlinear phase shift induced by the gain medium of the graphene-based waveguide. Therefore, the MI in each arm leads to form an ultrafast oscillations. Subsequently, the modulation bandwidth and modulation rate are influenced by the dynamical behavior of MI. 
The obtained results reveal that a modulation bandwidth of $\sim 81 \mathrm{GHz}$ at modulation speed of $\sim 2$ Tpps (pulse per second) is attainable. Even more, a modulation depth of $89 \%$ can be achieved. Meanwhile, the interference waveform unveils an amplification in the presence of the absolute MI. This can be perceived from Fig. 5(a) through which the modulation frequency approaches to the critical absolute MI frequency and the growing amplitude becomes more evident. Nonetheless, if SPPs damping is considered, the amplification in consequence of absolute MIcan just compensate the fast damping rate, still providing unpredictability to be useful for secure optical communication.

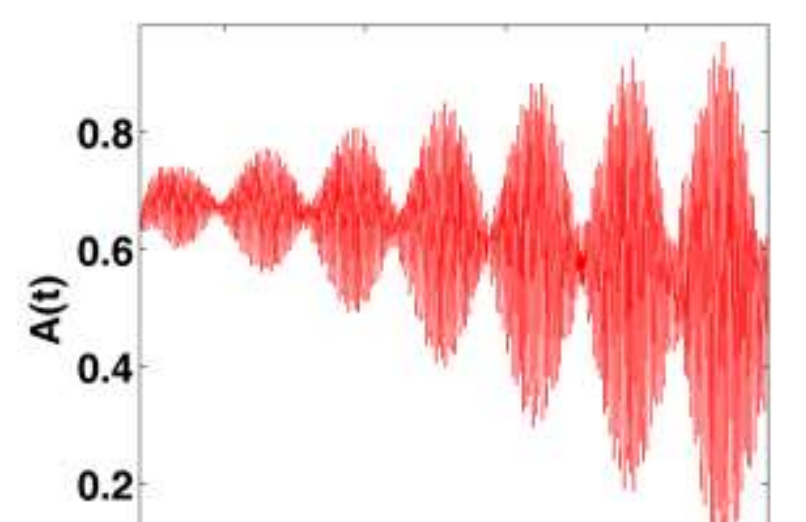

(a)

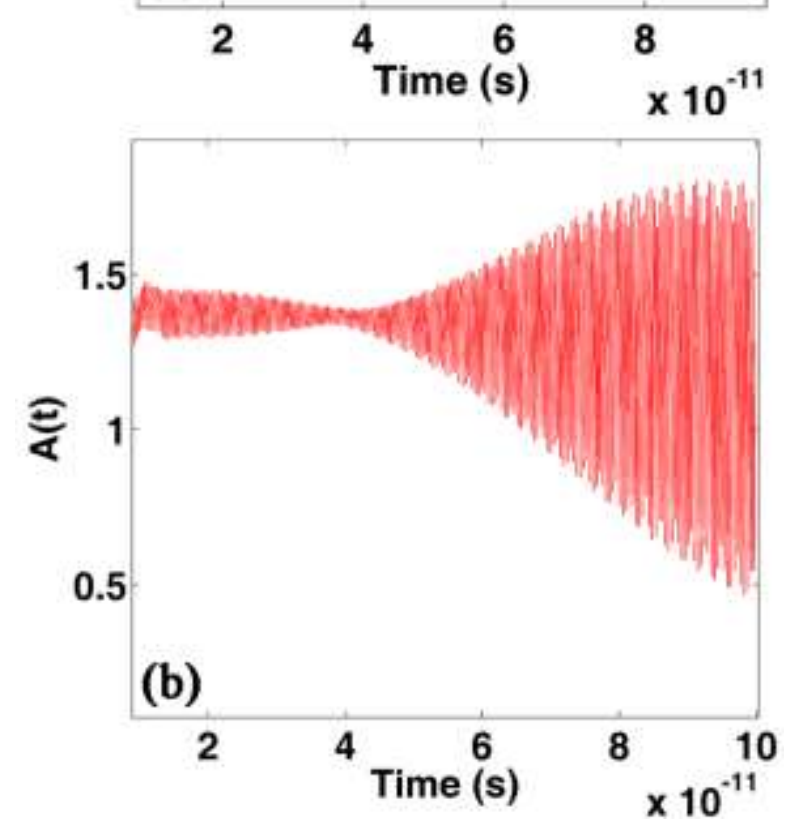

Fig. 5. Interference result for (a) case I, (b) case II.

The procedure is shown in Fig. 6 within a bifurcation diagram. The dashed line shows the threshold intensity above which the absolute MI and a transition to the chaotic regime will be dominant. To achieve it, a high feedback strength is required which cannot be simply fulfilled by an external mechanism (i.e. the Mach-Zehnder interferometer). In spite of this, for the assumed wavelength, the intraband transitions are allowed in graphene Dirac cone and thus, an internal feedback entity can be created by near field interactions of SPPs, increasing the total feedback strength [48].

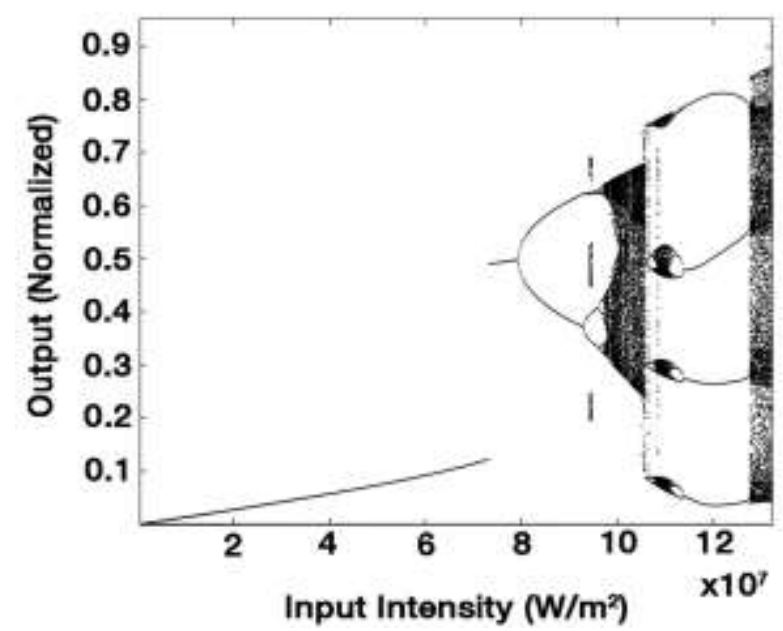

Fig. 6. Bifurcation diagram vs. the driving intensity

\section{B. Spatial MI and breather}

A uniform field distribution is necessary for a high performance modulation. Here, the nonlinear dynamics interfering in the modulation process may violate this demand. In fact, an absolute spatial MI regime causes the breather and rogue waves breeding thus, an irreversible instability [49-55]. In this connection, spatial amplitude profile is simulated in the proposed Mach-Zehnder modulator as shown in Fig.7. Although the waves propagating in both arms suffer the spatial MI, the waves interference appears more or less uniform in the output of the Mach-Zehnder interferometer for the case I (Fig. 7(a)). The situation is vice versa for the case II (Fig. 7(b)) in which the field fluctuations emerge more intense. 

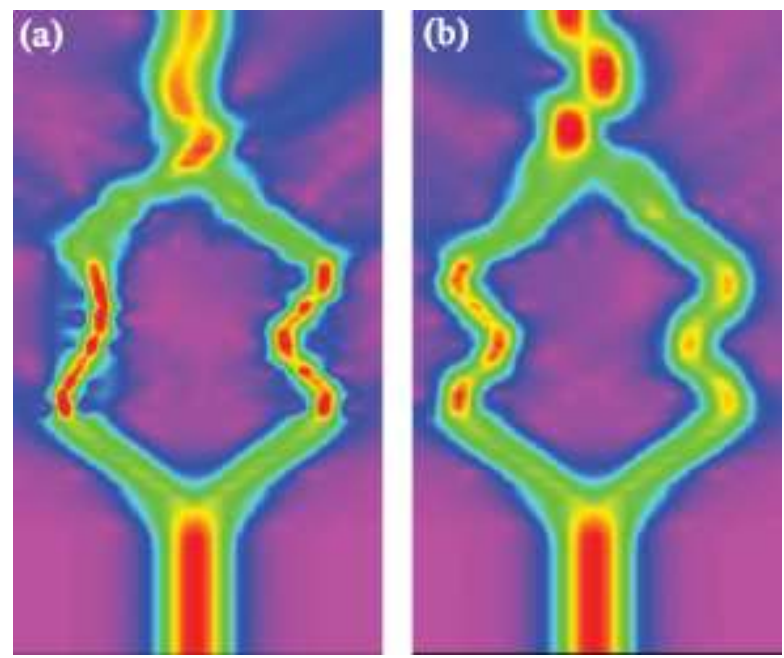

Fig. 7. Spatial amplitude obtained for (a) case I (b) case II of Mach-Zehnder interferometer.

Electric field effect resulting from the electrode structures my also impose a nonuniform field distribution - as it can be clearly seen from Fig. 7(a) and Fig. 7(b). Photoinduced doping of graphene-based waveguide may promise a more uniform profile, additionally bearing an energy saving [56-57].

\section{CONCLUSION}

A graphene-based all-optical SPPs modulator has been proposed using the MI. It has been shown that the nonlinear MI procedure can result in a broad band, high depth modulation with fast rate. The growing amplitude feature of MI also brings SPPs amplification which in turn can compensate the damping and consequently leads to long range SPPs. Absolute MI is a prior state to chaotic regime. Accordingly, the presented modulation approach can be used for secure optical communication due to the absolute MI unpredictable dynamical behavior.

\section{REFERENCES}

[1] R.W. Boyd, Nonlinear optics, Academic press, 2020.

[2] H. Gibbs, Optical bistability: controlling light with light, Elsevier, 2012.

[3] V.E. Zakharov and L.A. Ostrovsky, "Modulation instability: the beginning," Physica D, Vol. 238, pp. 540-548, 2009.
[4] V.E. Zakharov and A.A. Gelash, "Nonlinear stage of modulation instability," Phys. Rev. Lett. Vol. 111, pp. 054101 (1-5), 2013.

[5] M.A. Sharif, "Modulation instability of optical nonlinear media, a route to chaos," IEEE. Asia Communications and Photonics Conference and Exhibition (ACP), pp. 1-8, Nov. 2011.

[6] R.P. Sharma, K. Batra, and A.D. Verga, "Nonlinear evolution of the modulational instability and chaos using one-dimensional Zakharov equations and a simplified model," Phys. Plasmas, Vol. 12, pp. 022311 (1-7), 2005.

[7] M.A. Sharif, M. Borjkhani, and B. Ghafary, "Temporal modulation instability, transition to chaos in non-feedback biased photorefractive media," Opt. Commun. Vol. 319, pp. 17-24, 2014.

[8] J.M. Dudley, G. Genty, F. Dias, B. Kibler, and N. Akhmediev, "Modulation instability, Akhmediev Breathers and continuous wave supercontinuum generation," Opt. Express, Vol. 17, pp. 21497-21508, 2009.

[9] A. Demircan and U. Bandelow, "Supercontinuum generation by the modulation instability," Opt. Commun. Vol. 244, pp. 181-185, 2005.

[10]M. Conforti, A. Mussot, A. Kudlinski, and S. Trillo, "Modulational instability and pulse generation in dispersion oscillating fiber ring cavities," J. Opt. Soc. Am. Vol. 27, pp. NTh4A.3, 2014.

[11]D.Y. Tang, S. Fleming, W.S. Man, H.Y. Tam, and M.S. Demokan, "Subsideband generation and modulational instability lasing in a fiber soliton laser," J. Opt. Soc. Am. B, Vol. 18, pp. 1443-1450, 2011.

[12] S. Mosca, M. Parisi, I. Ricciardi, F. Leo, T. Hansson, M. Erkintalo, P. Maddaloni, P. De Natale, S. Wabnitz, and M. De Rosa, "Modulation instability induced frequency comb generation in a continuously pumped optical parametric oscillator," Phys. Rev. Lett, Vol. 121, pp. 093903 (1-19), 2018.

[13] R. Haldar, A. Roy, P. Mondal, V. Mishra, and S.K. Varshney, "Free-carrier-driven Kerr frequency comb in optical microcavities: Steady state, bistability, self-pulsation, and modulation instability," Phys. Rev. A, Vol. 99, pp. 033848 (1-14), 2019. 
[14] M.A. Sharif, "Modulation instability-enhanced frequency comb generation in graphene-based electro-optical modulator at terahertz frequency range," J. Opt. Vol. 22, pp. 095503 (1-9), 2020.

[15]P.T.S. DeVore, D. Borlaug, and B. Jalali, "Enhancing electrooptic modulators using modulation instability," Phys. status solidi RRL, Vol. 7, pp. 566-570, 2013.

[16]M. A. Sharif, B. Ghafary, and M.H.M. Ara, "A novel graphene-based electro-optical modulator using modulation instability," IEEE. Photon. Technol. Lett. Vol. 28, pp. 2897-2900, 2016.

[17]X. Du, I. Skachko, A. Barker, and E.Y. Andrei, "Approaching ballistic transport in suspended grapheme," Nat. Nanotechnol, Vol. 3, pp. 491-495, 2008.

[18] K.I. Bolotin, K.J. Sikes, Z. Jiang, M. Klima, G. Fudenberg, J. Hone, P. Kim, and H.L. Stormer, "Ultrahigh electron mobility in suspended grapheme," Solid state Commun, Vol. 146, pp. 351-355, 2008.

[19]H. Deng, F. Ye, B.A. Malomed, X. Chen, and N.C. Panoiu, "Optically and electrically tunable Dirac points and Zitterbewegung in graphene-based photonic superlattices," Phys. Rev. B, Vol. 91, pp. 201402 (1-5), 2015.

[20] M.F. Craciun, S. Russo, M. Yamamoto, and S. Tarucha, "Tuneable electronic properties in grapheme," Nano Today, Vol. 6, pp. 42-60, 2011.

[21]A. Ciattoni and C. Rizza, "Graphenenonlinearity unleashing at lasing threshold in graphene-assisted cavities," Phys. Rev. A, Vol. 91, pp. 053833 (1-7), 2015.

[22]N.A. Savostianova and S.A. Mikhailov, "Giant enhancement of the third harmonic in graphene integrated in a layered structure," Appl. Phys. Lett, Vol. 107, pp. 181104 (1-4), 2015.

[23]F. Shi, Y. Chen, P. Han, and P. Tassin - Small, "Broadband, Spectrally Flat, Graphene-based Terahertz Modulators," Small, Vol. 11, pp. 6044-6050, 2015.

[24] C.T. Phare, Y.H.D. Lee, J. Cardenas, and M. Lipson, "Graphene electro-optic modulator with $30 \mathrm{GHz}$ bandwidth," Nat. Photonics, Vol. 9, pp. 511-514, 2015.
[25] S. Luo, Y. Wang, X. Tong, and Z. Wang "Graphene-based optical modulators," Nanoscale Res. Lett. Vol. 10, pp. 1-11, 2015.

[26]B. Sensale-Rodriguez, R. Yan, M.M. Kelly, T. Fang, K. Tahy, W.S. Hwang, D. Jena, L. Liu, and H.G. Xing, "Broadband graphene terahertz modulators enabled by intraband transitions," Nat. Commun. Vol. 3, pp. 1-7, 2012.

[27]Ch. Ye, S. Khan, Zh. R. Li. E. Simsek, and V. $J$. Sorger, " $\lambda$-size ITO and graphene-based electro-optic modulators on SOI," IEEE. J. Sel. Top. Quantum Electron, Vol. 20, pp. 4049, 2014.

[28] Sh. Yu, X. Wu, K. Chen, B. Chen, X. Guo, D. Dai, L. Tong, W. Liu, and Y. Ron Shen "Alloptical graphene modulator based on optical Kerr phase shift," Optica, Vol. 3, pp. 541-544, 2016.

[29]D. Ansell1, I.P. Radko, Z. Han, F.J. Rodriguez, S.I. Bozhevolnyi, and A.N. Grigorenko, "Hybrid graphene plasmonic waveguide modulators," Nat. Commun. Vol. 6, pp. 1-6, 2015.

[30] X. Peng, R. Hao, Z. Ye, P. Qin, W. Chen, H. Chen, X. Jin, D. Yang, and E. Li, "Highly efficient graphene-on-gap modulator by employing the hybrid plasmonic effect," Opt. Lett. Vol. 42, pp.1736-1739, 2017.

[31]F. Zhou and W. Du, "Ultrafast all-optical plasmonic graphene modulator," Appl. Opt. Vol. 57, pp. 6645-6650, 2018.

[32]F. Sun, L. Xia, Ch. Nie, C. Qiu, L. Tang, J. Shen, T. Sun, L. Yu, P. Wu, Sh. Yin, Sh. Yan, and $\mathrm{Ch}$. Du "An all-optical modulator based on a graphene-plasmonic slot waveguide at 1550 nm," Appl. Phys. Express, Vol. 12, pp. 042009 (1-12), 2019.

[33]C. Haffner, W. Heni, Y. Fedoryshyn, J. Niegemann, A. Melikyan, D. L. Elder, B. Baeuerle, Y. Salamin, A. Josten, U. Koch, C. Hoessbacher, F. Ducry, L. Juchli, A. Emboras, D. Hillerkuss, M. Kohl, L. R. Dalton, C. Hafner, and J. Leuthold, "All-plasmonic Mach-Zehnder modulator enabling optical high-speed communication at the microscale," Nat. Photonics, Vol. 9, pp. 525-528, 2015.

[34]X. Guo, R. Liu, D. Hu, H. Hu, Zh. Wei, R.Wang, Y. Dai, Y. Cheng, K. Chen, K.Liu, G. Zhang, X. Zhu, Zh. Sun, X. Yang, and Q. Dai, "Efficient All-Optical Plasmonic 
Modulators with Atomically Thin Van Der Waals Heterostructures," Adv. Mater, Vol. 32, pp. 1907105 (1-8), 2020.

[35]H. Vahed and S.S. Ahmadi, "Hybrid plasmonic optical modulator based on multilayer grapheme," Opt. Quantum Electron. Vol. 52, pp. 1-2, 2020.

[36] J. Wang, X. Zhang, Y. Chen, Y. Geng, Y. Du, and $\mathrm{X}$. Li, "Design of a graphene-based silicon nitride multimode waveguide-integrated electro-optic modulator," Opt. Commun. Vol. 481, pp. 126531 (1-5), 2021.

[37]C. Ma, B. Xiao, D. Zhou, and L. Xiao, "A novel tunable terahertz wave modulator based on graphene and frequency selective surface (FSS)," Opt. Commun, Vol. 478, pp. 126375 (1-6), 2021.

[38] S. Wagner, C. Weisenstein, A.D. Smith, M. Östling, S. Kataria, and M.C. Lemme, "Graphene transfer methods for the fabrication of membrane-based NEMS devices," Microelectron. Eng, Vol. 159, pp. 108-113, 2016.

[39]M. Chen, R.C. Haddon, R. Yan, and E. Bekyarova, "Advances in transferring chemical vapour deposition graphene: a review," Mater Horizons, Vol. 4, pp. 10541063, 2017.

[40]H. Cheun Lee, W.-W. Liu, S.-P. Chai, A.R. Mohamed, A. Aziz, Ch.-S. Khe, N. M.S. Hidayah, and U. Hashim, "Review of the synthesis, transfer, characterization and growth mechanisms of single and multilayer grapheme," RSC Adv, Vol. 7, pp. 1564415693, 2017.

[41]A. Majumdar, J. Kim, J. Vuckovic, and F. Wang, "Electrical Control of Silicon Photonic Crystal Cavity by Graphene," Nano Lett. Vol. 13, pp. 515-518, 2013.

[42] S. Liu, P. Zhang, C. Lou, F. Xiao, and J. Zhao, "Numerical simulations of discrete propagations of light waves in optically induced planar waveguide arrays," J. Mod. Opt. Vol. 56, pp. 677-684, 2009.

[43] S.A. Mikhailov, "Quantum theory of the thirdorder nonlinear electrodynamic effects of graphene," Phys. Rev. B, Vol. 93, pp. 085403 (1-31), 2016.

[44] J.L. Cheng, N. Vermeulen, and J.E. Sipe, "Third order optical nonlinearity of graphene,"
New J. Phys. Vol. 16, pp. 053014 (1-17), 2014.

[45]E. Hendry, P.J. Hale, J. Moger, A.K. Savchenko, and S.A. Mikhailov, "Coherent Nonlinear Optical Response of Graphene," Phys. Rev. Lett. Vol. 105, pp. 097401 (1-4), 2010.

[46]V.E. Zakharov and L.A. Ostrovsky, "Modulation instability: the beginning," Physica D. Vol. 238, pp. 540-548, 2009.

[47] A.A. Balyakin and N.M. Ryskin, "A change in the character of modulation instability in the vicinity of a critical frequency," Tech. Phys. Lett. Vol. 30, pp. 175-177, 2004.

[48] M.A. Sharif, M. Khodavirdizadeh, S. Salmani, S. Mohajer, and M.H. MajlesAra, "Difference Frequency Generation-based ultralow threshold Optical Bistability in graphene at visible frequencies, an experimental realization," J. Mol. Liq, Vol. 284, pp. 92-101, 2019.

[49]M.A. Alejo, L. Fanelli, and C. Muñoz, "Review on the Stability of the Peregrine and Related Breathers," Front. Phys. Vol. 8, pp. 404 (1-8), 2020.

[50] J.M. Dudley, F. Dias, M. Erkintalo, and G. Genty, "Instabilities, breathers and rogue waves in optics," Nat. Photonics, Vol. 8, pp. 755-764, 2014.

[51]G. Mu, Z. Qin, and R. Grimshaw, "Dynamics of rogue waves on a multisoliton background in a vector nonlinear Schrodinger equation," SIAM J. Appl. Math. Vol. 75, pp. 1-20, 2015.

[52]B.F. Feng, L. Ling, and D.A. Takahashi, "Multi-breather and high-order rogue waves for the nonlinear Schrödinger equation on the elliptic function background," Stud. Appl. Math. Vol. 144, pp.46-101, 2020.

[53]L.L. Feng and T.T. Zhang, "Breather wave, rogue wave and solitary wave solutions of a coupled nonlinear Schrödinger equation," Appl. Math. Lett. Vol. 78, pp. 133-140, 2018.

[54]G.T. Adamashvili and D.J. Kaup, "Optical surface breather in graphene," Phys. Rev. A, Vol. 95, pp.053801, 2017.

[55]M.A. Sharif, "Spatio-temporal modulation instability of surface plasmon polaritons in graphene-dielectric heterostructure," Physica E Low Dimens. Syst. Nanostruct. Vol. 105, pp.174-181, 2019. 
[56]P.- H. Ho, Ch.- H. Chen, F.- Y. Shih, Y.- R. Chang, Sh.- S. Li, W.- H. Wang, M.- Ch. Shih, W.- T. Chen, Y- P. Chiu, M.- K. Li, Y- S. Shih, and Ch.- W. Chen, "Precisely Controlled Ultrastrong Photoinduced Doping at Graphene-Heterostructures Assisted by Trap-State-Mediated Charge Transfer," Adv Mater. Vol. 27, pp. 7809-7815, 2015.

[57]L. Misseeuw, T. Ciuk, A. Krajewsk, I. Pasternak, W. Strupinski, B. Feigel, M. Khoder, I. Vandriessche, J. Van Erps, S.Van Vlierberghe, H. Thienpont, P. Dubruel, and N. Vermeulen, "Localized optical-quality doping of graphene on silicon waveguides through a TFSA-containing polymer matrix," J. Mater. Chem. C, Vol. 6, pp. 10739-10750, 2018.

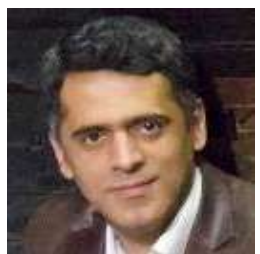

Morteza Abdolahi Sharif has worked since 2010 as a faculty member in Optics and laser Engineering Group of Urmia University of Technology (UUT). His current academic degree is assistant professor. He is also the head of Photonics Lab in UUT. He has received his $\mathrm{Ph} . \mathrm{D}$. in Iran University of Science and Technology on the nonlinear optical properties of nanostructures and nanomaterials. He has particularly working on graphene and the graphene-based devices and waveguides. His other working fields are plasmonics, optical communication and chaos. 
THIS PAGE IS INTENTIONALLY LEFT BLANK. 